\title{
Agente de Suporte à Decisão Multicritério com Soma Ponderada-Fuzzy em Gestão Pública Participativa: Um Estudo de Caso em Gestão Ambiental
}

\author{
Leonardo Afonso Amorim ${ }^{1}$, Vinícius Sebba Patto ${ }^{1}$, Luís Alfredo Costa Freitas ${ }^{2}$, \\ Iwens Gervásio Sene Junior ${ }^{1}$, Renato de Freitas Bulcão Neto ${ }^{1}$ \\ ${ }^{1}$ Instituto de Informática - Universidade Federal de Goiás (UFG) \\ Caixa Postal 131 - 74.001-970 \\ Goiânia - GO - Brasil \\ ${ }^{2}$ Instituto Chico Mendes de Conservação da Biodiversidade - ICMBio \\ Rua 229, n 95, Setor Leste Universitário - 74.605-090 \\ Goiânia - GO - Brasil \\ leonardoamorimeinf.ufg.br ${ }^{1}$, viniciussebbadinf.ufg.br ${ }^{1}$ \\ luis.freitas@icmbio.gov.br ${ }^{2}$ \\ iwenseinf.ufg.br ${ }^{1}$, renatodinf.ufg.br ${ }^{1}$
}

\begin{abstract}
This paper presents a decision support agent (DSA) for participatory public management. Facing environmental management challenges and the importance of the environment, a case study was performed to analyze DSA's outcomes, whose profile is more sensitive to environmental concerns providing the environmental manager a natural language interface with Fuzzy logic for assigning weights to the environmental aspects. The main goal of this paper is to demonstrate the importance of changing the method of assigning weights in the weighted sum by Fuzzy logic because the human being is more conditioned to rank problems out in natural language. Experiments demonstrated the use of fuzzy improves usability and, consequently, the effectiveness of DSA.
\end{abstract}

Keywords: decision support, fuzzy logic, multi-criteria analysis, participatory public management, environmental management.

Resumo. Este artigo apresenta um agente para suporte à decisão (ASD) em gestão pública participativa. Face aos desafios da gestão ambiental, foi proposto um estudo de caso para analisar as saídas do ASD, com perfil mais sensível às preocupações ambientais, disponibilizando ao gestor ambiental uma interface em linguagem natural com Lógica Fuzzy para atribuição de pesos aos aspectos ambientais. O principal objetivo deste artigo é demonstrar a importância de alterar o método de atribuição de pesos na Soma Ponderada por meio da Lógica Fuzzy, visto que o ser humano está mais condicionado a ranquear problemas em linguagem natural. Experimentos demonstraram que o uso de Fuzzy melhora a usabilidade e, consequentemente, a eficácia do ASD, pois geram ranqueamentos mais próximos ao ideal, segundo a visão de um gestor ambiental.

Palavras-chave: suporte à decisão, lógica fuzzy, análise multicritério, gerenciamento público participativo, gestão ambiental. 


\section{Introdução}

A gestão pública, que em geral é uma tarefa complexa, é de grande importância na manutenção do meio ambiente e exige abordagem sistêmica acerca de vários aspectos [Machado Junior et al. 2013]. Segundo [Afonso Amorim et al. 2014] e [Gamper and Turcanu 2007], é importante considerar que os elementos ambientais estão correlacionados e sem gestão pública adequada para controlar o impacto ambiental causado pela exploração desses recursos, há considerável risco de redução da sua disponibilidade. Consequentemente, aumenta-se a dificuldade de exploração, que por sua vez, aumenta o uso de recursos naturais. Adicionalmente, a redução de recursos naturais pode afetar a perpetuação de espécies vegetais ou animais. Além disso, a sua exploração pode afetar a qualidade do ar, que por sua vez, pode afetar a temperatura média global.

Atualmente, tomadas de decisão no setor público não devem considerar apenas aspectos financeiros, como análise de custo-benefício e análise de custo eficácia. É essencial para a construção de uma gestão pública incluir aspectos ambientais, que geralmente entram em conflito com os aspectos econômicos. Nesse contexto, o emprego da análise multicritério tem ganhado importância quando os critérios a analisar não podem ser expressos facilmente em termos financeiros [Gamper and Turcanu 2007].

Em [Afonso Amorim et al. 2014], é apresentado um Agente de Suporte à Decisão (ASD) que faz uso de técnicas de mineração de dados e análise multicritério para apontar problemas relevantes. Para facilitar a compreensão do trabalho, apresenta-se o contexto em que este se insere: o projeto ADGEPA (Assistente Digital para Gestão Pública Participativa), com os seus módulos e um modelo de simulação multiagentes. Os objetivos do ADGEPA incluem criar um canal de comunicação entre cidadão e gestor público, auxiliar o gestor na tomada de decisão e promover gestão pública participativa pela inserção da maioria dos anseios populares em tomadas de decisão.

Uma das grandes dificuldades do gestor público é ponderar numericamente quais são os aspectos socioambientais mais importantes. Em [Afonso Amorim et al. 2014], o gestor público define pesos para cada aspecto socioambiental (água, ar, solo, desmatamento, invasão social, segurança, educação, saúde, infraestrutura urbana, invasão social) para que seja aplicado o método multicritério Soma Ponderada e, por fim, seja gerado um ranqueamento dos aspectos com maior prioridade. Segundo [Rowley et al. 2012], acredita-se que a definição de peso numérico, quando se analisa diversos critérios, tende a ser subjetivo, portanto o mesmo poderia variar de acordo com cada gestor, e alguns na dificuldade de defini-lo podem escolher qualquer valor.

Uma possibilidade para dar mais acurácia e melhorar a usabilidade do gestor público na atribuição de pesos é usar Lógica Fuzzy em conjunto com o método multicritério Soma Ponderada, pois o ser humano está mais condicionado a classificar problemas em linguagem natural [Yeh and Xu 2013]. Com o uso da Lógica Fuzzy, é possível fazer inferência por meio de conceitos linguísticos imprecisos e com certo grau de ambiguidade, de forma análoga àquela que os seres humanos fazem no mundo real. Os conjuntos nebulosos são uma forma de representar imprecisões encontradas, as quais os seres humanos tratam com grande habilidade, normalmente existentes em problemas reais, os quais os conjuntos tradicionais não podem representar adequadamente.

Portanto, o objetivo deste trabalho é melhorar a atribuição de pesos no método 
Soma Ponderada, que é um dos métodos utilizados pelo o ASD. Ao invés de definir que o peso para o indicador X é 0,14 é mais natural para o gestor definir em termos linguísticos que um atributo ecológico tem uma determinada característica classificada como muito baixa, baixa, média ou alta.

Os benefícios esperados com a aplicação de termos linguísticos incluem a melhora da usabilidade do método de suporte à decisão, para que o gestor possa atribuir pesos mais adequados aos indicadores socioambientais, e o aumento da acurácia do ranqueamento gerado pelo ASD, pois os pesos serão gerados com menor grau de incerteza.

Este artigo está assim organizado: a Seção 2 apresenta trabalhos relacionados, o projeto ADGEPA, os desafios e a motivação para este trabalho; a Seção 3 descreve o ASD, seu processo de mineração de dados e sua racionalidade; a Seção 4 detalha a modelagem fuzzy do problema; as Seções 5 e 6 descrevem, respectivamente, os experimentos realizados e uma discussão sobre os resultados obtidos; e a Seção 7 resume as conclusões deste trabalho.

\section{Trabalhos correlatos}

A análise multicritério com Lógica Fuzzy tem sido bem empregada em gestão pública de forma a destacar a importância dos aspectos ambientais.

Em [Yeh and Xu 2013] é utilizado o método de suporte à decisão fuzzy para elaborar um planejamento sustentável para o problema de lixos eletrônicos. Nesse trabalho, uma série de modelos foi desenvolvida para determinar pesos otimizados para três dimensões de sustentabilidade (ambiental, econômica e social) para aplicação do método multicritério AHP (Analytic Hierarchy Process).

Na pesquisa de [Chung and Kim 2014] é proposto o método fuzzy TOPSIS (Technique for Order of Preference by Similarity to Ideal Solution) para desenvolvimento de abordagem multicritério com Lógica Fuzzy para priorizar locais de utilização de águas residuais tratadas considerando cenários de mudanças climáticas. A hibridização da Lógica Fuzzy com o TOPSIS tem como objetivo neste trabalho a priorização final das alternativas como método primário para superar incertezas relacionadas aos valores de ponderação e dados de entrada para tomada de decisão.

No trabalho de [Rowley et al. 2012] são analisados métodos multicritério como Soma Ponderada, AHP, SMART, TOPSIS, MACBETH, ELECTRE, PROMETHEE quando aplicados em problemas ambientais que envolvam sustentabilidade. Esse trabalho ressalta a importância de saber escolher corretamente o método multicritério, pois uma escolha errada pode comprometer a definição de pesos e agregação, introduzindo subjetividade e incerteza, e que a Soma Ponderada é usada para formular um julgamento abrangente sobre o desempenho de sustentabilidade em relação às alternativas.

Já em [Wibowo and Deng 2013] é proposto um framework para melhorar a eficácia do processo de construção de consenso para tomada de decisão multicritério. Ele usa números fuzzy para modelar a subjetividade e imprecisão das decisões humanas.

O ADGEPA é um projeto inovador para suporte à tomada de decisão participativa em gestão pública. Este projeto não se preocupa em determinar valores monetários para investimentos, apenas identificar e ranquear os aspectos mais significantes para mitigar os problemas socioambientais mais relevantes aos seus usuários [Lima Silva et al. 2013]. 
Os trabalhos relacionados supracitados investigaram diversos aspectos importantes como suporte à decisão com ranqueamento de alternativas, gestão e aprendizagem humana em gerenciamento de recursos. Todavia, até onde os autores deste trabalho investigaram, não foi encontrado projeto que integrasse suporte à decisão em gestão pública participativa ${ }^{1}$ que forneça ajustes de pesos de critérios em linguagem natural com correlações espaciais, temporais e de aspectos socioambientais em centros urbanos para promover uso mais eficiente de recursos públicos financeiros.

Embora haja uma grande quantidade de artigos do método AHP com Lógica Fuzzy, o método multicritério Soma Ponderada foi mantido pelos seguintes motivos: facilidade de implementação, não é necessário fazer comparação par a par entre critérios, facilidade para estruturar o problema e fácil entendimento o que proporciona transparência. O ASD do projeto ADGEPA tem papel primordial, haja vista que ele faz busca de correlação entre denúncias, locais e momentos; em seguida, ele faz análise multicritério para ranquear os problemas socioambientais mais relevantes aos usuários e que são ao mesmo tempo mais relacionados à ocorrência de outros problemas (detalhes na Seção 3).

Com auxílio de especialista do domínio foram selecionados 23 aspectos ambientais (que incluem água, ar, solo, uso ilegal de recursos naturais e invasão ilegal de áreas protegidas) e 37 aspectos sociais (que abarcam segurança pública, educação, saúde pública, infraestrutura urbana e invasão ilegal de áreas e prédios públicos) para a aplicação web e quatro variáveis atmosféricas (umidade relativa do ar, luminosidade, temperatura e monóxido de carbono) para a rede de sensores. O ASD busca fazer correlação entre esses aspectos, o tempo e locais de ocorrência [Afonso Amorim et al. 2014].

\section{Agente de Suporte a Decisão}

O ADGEPA é implementado como um sistema web, que pode ser acessado por meio de smartphones ou computadores desktop, no qual uma denúncia é composta por coordenadas geográficas, aspecto socioambiental, momento da denúncia e o id do usuário. A primeira atividade do ASD é agrupar denúncias de acordo com coordenadas (isso define microrregiões em um mapa). Em seguida, esses grupos são reagrupados por intervalos temporais. Assim, cada novo grupo significa um conjunto de denúncias em uma localidade muito próxima e em momento muito próximo - isso representa um registro. A saída de dados é um arquivo do tipo $C S V$ em que todos os aspectos socioambientais são considerados como campos.

Nos experimentos iniciais, foram geradas 100.000 denúncias $^{2}$ em uma região metropolitana. Foi considerado um intervalo temporal de 30 dias. $\mathrm{O}$ arquivo $C S V$ foi então usado como entrada para o ASD, que por sua vez, com o uso da API do WEKA, gerou 100 agrupamentos (doravante clusters) espaciais e, a partir de cada um dos clusters, gerou 10 novos clusters temporais (ambos com o algoritmo K-means). Em seguida, com os 1000 clusters espaço-temporais, foi montado um Data Warehouse (cada um dos

\footnotetext{
${ }^{1}$ Gestão Pública Participativa é uma gestão em que membros da sociedade civil e representantes de classes são convidados a participar dos processos de tomada decisão.

${ }^{2}$ As denúncias foram geradas por meio de um programa desenvolvido pelos autores. Futuramente, pretende-se usar Simulação Multiagentes para a geração de denúncias uma vez que a aplicação web do ADGEPA ainda não está disponível.
} 
clusters equivale a um registro). Assim, cada registro apresenta a quantidade que cada aspecto foi denunciado em uma área muito pequena e um intervalo de tempo muito curto.

O objetivo da clusterização temporal é fazer com que as denúncias não se tornem obsoletas com o passar do tempo, enquanto que a clusterização espacial foi feita para limitar o espaço geográfico visando aumentar a probabilidade das denúncias terem relação entre si. Na Tabela 1, é possível observar um trecho de uma amostra de cluster temporal. As colunas da Tabela 1 representam em sequência:

1. ID: Número de identificação do usuário;

2. IND: Número que representa o indicador;

3. ANO: Ano da denúncia;

4. MÊS: Mês da denúncia;

5. SE: Semana da denúncia;

6. DIA: Dia da denúncia;

7. H: Hora da denúncia;

8. M: Minuto da denúncia;

9. S: Segundo da denúncia;

10. COORD.X: Coordenada $X$ do local da denúncia;

11. COORD.Y: Coordenada Y do local da denúncia;

12. CLUS: Número do cluster temporal.

Tabela 1. Trecho da Amostra do Primeiro Cluster Temporal

\begin{tabular}{|c|c|c|c|c|c|c|c|c|c|c|c|}
\hline ID & IND & ANO & MÊS & SE & DIA & H & M & S & COO. X & COO. Y & CLUS \\
\hline 41 & 49 & 2013 & 6 & 23 & 6 & 4 & 41 & 41 & -49.245265 & -16.692522 & 0 \\
\hline 80 & 44 & 2013 & 6 & 23 & 6 & 7 & 56 & 14 & -49.245422 & -16.693608 & 0 \\
\hline 69 & 28 & 2013 & 6 & 23 & 5 & 7 & 55 & 1 & -49.244687 & -16.691446 & 0 \\
\hline 24 & 26 & 2013 & 6 & 23 & 5 & 6 & 5 & 50 & -49.24415 & -16.69297 & 0 \\
\hline 6 & 36 & 2013 & 6 & 23 & 6 & 2 & 52 & 9 & -49.242085 & -16.692458 & 0 \\
\hline 23 & 21 & 2013 & 6 & 23 & 6 & 21 & 37 & 50 & -49.242882 & -16.6927 & 0 \\
\hline 61 & 49 & 2013 & 6 & 23 & 5 & 22 & 29 & 26 & -49.243431 & -16.692206 & 0 \\
\hline 44 & 5 & 2013 & 6 & 23 & 5 & 13 & 59 & 24 & -49.242911 & -16.6928 & 0 \\
\hline 46 & 15 & 2013 & 6 & 23 & 5 & 15 & 33 & 28 & -49.245974 & -16.693439 & 0 \\
\hline$\ldots$ & $\ldots$ & $\ldots$ & $\ldots$ & $\ldots$ & $\ldots$ & $\ldots$ & $\ldots$ & $\ldots$ & $\ldots$ & & $\ldots$ \\
\hline 92 & 44 & 2013 & 6 & 23 & 6 & 13 & 37 & 42 & -49.24651 & -16.692787 & 0 \\
\hline 49 & 50 & 2013 & 6 & 23 & 5 & 6 & 46 & 2 & -49.243608 & -16.692815 & 0 \\
\hline 16 & 7 & 2013 & 6 & 23 & 5 & 17 & 45 & 37 & -49.243369 & -16.693526 & 0 \\
\hline
\end{tabular}

Em seguida, considera-se o suporte para ranquear os 10 aspectos mais denunciados (valor arbitrário). Em mineração de dados, interpreta-se suporte como a probabilidade de um elemento ocorrer em uma coleção de registros [Larose 2004]. O cálculo do suporte é definido pela Equação 1. Neste trabalho foram definidos que seriam analisados 10 aspectos, mas a quantidade pode ser definida pelo gestor de acordo com seus objetivos.

$$
\operatorname{supp}(X)=\frac{\text { registros que possuem } X}{\text { total de registros }}
$$


A próxima etapa consiste em criar a matriz de decisão. A matriz é construida baseada no conceito de confiança. Em mineração de dados, interpreta-se confiança como a probabilidade de um elemento acontecer, dado que outro elemento também ocorra em mesmos registros [Larose 2004]. Para isso, o ASD encontra a média das confianças entre cada um dos 10 aspectos com maior suporte e os aspectos dos 10 grupos de aspectos socioambientais $^{3}$. O cálculo da confiança é definido pela Equação 2. O resultado deste processo inicial do ASD é uma matriz de dimensão 10 x 10[Afonso Amorim et al. 2014].

$$
\operatorname{conf}(X \Rightarrow Y)=\frac{\operatorname{supp}(X \cup Y)}{\operatorname{supp}(Y)}
$$

Após gerar a matriz de decisão, o ASD aplica uma técnica de análise multicritério. O primeiro protótipo do ASD foi implementado para usar o Método Multicritério de Soma Ponderada que, segundo [Almeida et al. 2012], é composto dos seguintes passos:

1. somar os elementos das linhas;

2. normalizar os elementos da linha pelo resultado da soma da linha:

$$
u_{i j}=\frac{a_{i j}}{\sum_{j=0}^{n} a_{i j}}, \forall j \in[1, n]
$$

No qual:

- $i$ indica a linha da matriz (foram escolhidos os 10 indicadores de maior suporte);

- $j$ indica a coluna da matriz (há 10 grupos de aspectos, logo 10 colunas e $j$ vai de 0 a 9);

- $a$ é o valor da média das confianças entre o aspecto e os aspectos de um grupo; e

- $u$ é o valor da média das confianças normalizado para cada elemento da matriz.

3. aplicar pesos do ASD, que variam de acordo com o perfil;

4. fazer a soma dos elementos das linha para identificar a ordem dos aspectos mais citados pelos usuários (maior suporte) e que possuem maior associação (confiança) com grupos de aspectos socioambientais de maior importância para o ASD.

Pode-se calcular os dois últimos passos da seguinte maneira:

$$
R\left(u_{i}\right)=\sum_{j}^{n} w_{j} a_{i j}, \forall i \in[1, n]
$$

Em que:

- $i$ indica a linha da matriz;

- $j$ indica a coluna da matriz, que vai de 0 a $n$ (neste trabalho, de 0 a 9);

- $w$ indica o peso aplicado ao grupo de indicadores;

- $u$ é o valor normalizado da média das confianças entre o aspecto e os aspectos de um grupo; e

- $R$ é o resultado do somatório para cada linha.

\footnotetext{
${ }^{3}$ Os 10 grupos são: água, ar, solo, uso ilegal de recursos naturais (áreas verdes), invasão ilegal de áreas protegidas (áreas verdes), segurança pública, educação, saúde pública, infraestrutura urbana e invasão ilegal de áreas e prédios públicos. Cada grupo possui no mínimo um aspecto, ou social, ou ambiental.
} 


\section{Problema}

Para trabalhar com Lógica Fuzzy o primeiro passo é definir quais são as variáveis existentes para criar posteriormente as regras com o auxílio do gestor ambiental. Os atributos ecológicos de acordo com [Tulloch et al. 2015] e [Salafsky et al. 2013] que devem ser avaliados são: áreas verdes (desmatamento e invasão ambiental), água, solo e ar. Cada atributo deste pode ser classificado de acordo com as seguintes variáveis: resiliência, abrangência da ameaça, severidade e irreversibilidade [Tulloch et al. 2015] e [Salafsky et al. 2013]. Resiliência refere-se à estabilidade ambiental do atributo ecológico ou à saúde do mesmo, avaliada em função da possibilidade de ser restaurado após a retirada da ameaça. Ela pode ser classificada como:

- Muito baixa: quando é praticamente impossível reverter os efeitos da ameaça sobre o atributo ecológico, e pouco provável que o bem estar socioambiental que dependa do atributo ecológico possa ser restaurado;

- Baixa: quando é possível tecnicamente reverter os efeitos da ameaça ao atributo ecológico e restaurar o bem estar socioambiental que dependa do atributo;

- Média: quando os efeitos da ameaça sobre o atributo ecológico podem ser revertidos e o bem estar socioambiental pode ser restaurado com recursos razoáveis;

- Alta: quando os efeitos da ameaça sobre o atributo ecológico podem ser facilmente revertidos e o bem estar socioambiental restaurado a um custo relativamente baixo;

Após isso, deve-se verificar a criticidade ou o grau da ameaça. Ameaça é uma atividade humana que afeta negativamente o alvo de conservação. Ameaça crítica é uma ameaça direta que foi priorizada como mais importante, de acordo com sua abrangência. Abrangência é a proporção do alvo que se acredita que será afetada de alguma forma pela ameaça, dado a continuidade das circunstâncias e tendências atuais.

- Muito Alta: quando a ameaça tende a ser predominante, afetando o alvo de conservação no todo ou na maioria (71 a $100 \%$ da população);

- Alta: quando a ameaça tende a ser abrangente, afetando o alvo de conservação em grande parte (31 a $70 \%$ da população);

- Média: quando a ameaça tende a ser restrita, afetando uma parte do alvo de conservação (11 a 30\% da população);

- Baixa: quando a ameaça tende a ser muito restrita, afetando uma pequena parcela do alvo de conservação (1 a 10\% da população);

Severidade refere-se ao nível de dano ao alvo causado pela ameaça, que pode ser razoavelmente esperado, dado a continuidade das circunstâncias e tendências atuais e pode ser classificada como:

- Muito Alto: quando a ameaça tende a suprimir ou prejudicar gravemente o bem-estar socioambiental da população afetada;

- Alto: quando a ameaça tende a prejudicar seriamente o bem-estar socioambiental da população afetada;

- Médio: quando a ameaça tende a prejudicar razoavelmente o bem-estar socioambiental da população afetada;

- Baixo: quando a ameaça tende a prejudicar somente um pouco a população afetada; 
Irreversibilidade refere-se ao grau em que os efeitos de uma ameaça possam ser revertidos e o alvo afetado pode ser restaurado, após a eliminação da ameaça e pode ser classificada como:

- Muito Alta: quando é impossível reverter os efeitos da ameaça e pouco provável que o bem estar socioambiental possa ser restaurado;

- Alta: quando é possível tecnicamente reverter os efeitos da ameaça e restaurar o bem estar socioambiental;

- Média: quando os efeitos da ameaça podem ser revertidos e o bem estar socioambiental pode ser restaurado com recursos razoáveis;

- Baixa: quando os efeitos da ameaça podem ser facilmente revertidos e o bem estar socioambiental restaurado a um custo relativamente baixo;

Para a geração de pesos de acordo com a combinação das variáveis foram usadas as seguintes regras construídas por meio de pesquisa realizada com 10 analistas ambientais do ICMBio e Ibama:

1. Se Resiliência(Alta) e Abrangência(Baixa) e Severidade(Baixa) e Irreversibilidade(Baixa), então o peso será igual a 0.12 (Situação não crítica);

2. Se Resiliência(Média) e Abrangência(Média) e Severidade(Média) e Irreversibilidade(Média), então o peso será igual a 0.14 (Situação crítica mas aceitável);

3. Se Resiliência(Baixa) e Abrangência(Alta) e Severidade(Alta) e Irreversibilidade(Alta), então o peso será igual a 0.16 (Situação crítica e abaixo do aceitável);

4. Se Resiliência(Muito baixa) e Abrangência(Muito Alta) e Severidade(Muito Alta) e Irreversibilidade(Muito Alta), então o peso será igual a 0.18 (Situação muito crítica e inaceitável);

Portanto, as regras fuzzy foram definidas para gerar os seguintes pesos para $w$ : $0.18,0.16,0.14$ e 0.12 para os aspectos ambientais. Como o perfil do gestor é ambiental, atribuiu-se $60 \%$ do peso total para os indicadores ambientais. Logo, os pesos definidos anteriormente somados equivalem a 0.60 . Os $40 \%$ restantes são divididos igualmente entre os indicadores sociais, isto é, 0.08 para cada um.

A distribuição dos pesos para os aspectos socioambientais consiste, primeiramente, em verificar a quais grupos cada aspecto socioambiental pertence. Isso é fundamental pois determinará qual será o peso para cada aspecto. Após isso, é atribuído um peso para cada aspecto de acordo com sua importância. Por exemplo, se o atributo socioambiental pertencer ao grupo de áreas verdes (dividido em dois subgrupos: desmatamento e invasão ambiental), ele terá um peso maior que grupos de água, solo e ar respectivamente. Após esta verificação, soma-se o valor total dos pesos atribuídos aos aspectos ambientais e subtrai este valor por $\mathrm{um}^{4}$. O resultado desta subtração é dividido pela quantidade de aspectos sociais, portanto os pesos para os aspectos sociais serão iguais. Assim, o perfil do gestor será ambiental.

O controlador usado neste experimento foi o Sugeno que consiste numa simplificação do controlador de $\mathrm{Mamdani}^{5}$, onde o conseqüiente de cada regra é definido

\footnotetext{
${ }^{4}$ A soma dos pesos na Soma Ponderada deve ser igual a 1.

${ }^{5}$ Sugeno e Mamdani são métodos de inferência simples e eficientes que podem ser usados pela ferramenta Fuzzy Logic toolbox do MATLAB.
} 
como uma função das variáveis lingüísticas de entrada [Ying et al. 1999]. Isto é, a regra geral $R_{i}$ pode ser escrita como: Regra $\left(\mathrm{R}_{i}\right)$ : se $x$ é $A_{i}$ e $y$ é $B_{i}$ então $z=f_{i}(x, y)$. O resultado de cada regra é, portanto, um valor numérico (não um conjunto fuzzy), que assume como peso o valor da pertinência resultante do processamento do antecedente da regra. Essa determinação dispensa, portanto, a definição de uma função de implicação específica.

A resposta final do controlador é obtida pela média ponderada das respostas das regras individuais. Isto é, neste tipo de controlador não cabe processo de defuzificação. $O$ valor de $z$ pode também ser definido como um valor constante, que pode ser interpretado como um conjunto fuzzy com a característica especial de apresentar um único valor com pertinência igual a um e todos os demais com pertinência zero [Ying 2000]. Os valores das variáveis linguísticas foram definidas por meio de números fuzzy triangulares.

\section{Experimentação}

Dois experimentos foram realizados com o objetivo verificar a acurácia e a usabilidade do método com Lógica Fuzzy.

\subsection{Experimento 1}

Este experimento consiste em comparar os ranqueamentos gerados pela Soma Ponderada sem Fuzzy e a Soma Ponderada-Fuzzy, com um ranqueamento ideal de acordo com o gestor ambiental. O objetivo é verificar qual método produziu um ranqueamento mais próximo do ideal. A Tabela 2 mostra o ranqueamento ideal do ponto de vista do gestor ambiental, que sabe que cada aspecto socioambiental pode e deve ter pesos que impliquem em uma melhor colocação no ranqueamento de prioridades da gestão ambiental.

Tabela 2. Ranking ideal de aspectos socioambientais segundo gestor ambiental

\begin{tabular}{|c|c|}
\hline Ordem & Aspecto socioambiental \\
\hline 1 & Áreas verdes: Extração ou exploração de recursos naturais \\
\hline 2 & Áreas verdes: Pesca irregular \\
\hline 3 & Solo: Extração irregular de areia, argila ou cascalho \\
\hline 4 & Ar: Emissão de odores fétidos \\
\hline 5 & Falta de creche \\
\hline 6 & Invasão de terreno público \\
\hline 7 & Roubo \\
\hline 8 & Inexistência de bueiros \\
\hline 9 & Traficantes \\
\hline 10 & Falta de leitos em hospitais \\
\hline
\end{tabular}

A Tabela 3 mostra o ranqueamento gerado por meio do método multicritério Soma Ponderada sem aplicação de Fuzzy para geração de pesos: 
Tabela 3. Ranking de aspectos socioambientais sem Fuzzy

\begin{tabular}{|c|c|}
\hline Ordem & Aspecto socioambiental \\
\hline 1 & Ar: Emissão de odores fétidos \\
\hline 2 & Áreas verdes: Extração ou exploração de recursos naturais \\
\hline 3 & Solo: Extração irregular de areia, argila ou cascalho \\
\hline 4 & Âreas verdes: Pesca irregular \\
\hline 5 & Falta de creche \\
\hline 6 & Invasão de terreno público \\
\hline 7 & Roubo \\
\hline 8 & Inexistência de bueiros \\
\hline 9 & Traficantes \\
\hline 10 & Falta de leitos em hospitais \\
\hline
\end{tabular}

A Tabela 4 mostra o ranqueamento gerado por meio do método multicritério Soma Ponderada com aplicação de Fuzzy para geração de pesos:

Tabela 4. Ranking de aspectos socioambientais com Fuzzy

\begin{tabular}{|c|c|}
\hline Ordem & Aspecto socioambiental \\
\hline 1 & Áreas verdes: Extração ou exploração de recursos naturais \\
\hline 2 & Áreas verdes: Pesca irregular \\
\hline 3 & Ar: Emissão de odores fétidos \\
\hline 4 & Solo: Extração irregular de areia, argila ou cascalho \\
\hline 5 & Falta de creche \\
\hline 6 & Invasão de terreno público \\
\hline 7 & Roubo \\
\hline 8 & Inexistência de bueiros \\
\hline 9 & Traficantes \\
\hline 10 & Falta de leitos em hospitais \\
\hline
\end{tabular}

Os resultados mostram que a Lógica Fuzzy influencia o ranqueamento dos aspectos socioambientais. Por meio do método Spearman Ranking Correlation (SRC) [Gauthier 2001] é possível afirmar que o ranqueamento com Fuzzy (Tabela 4) tem 18\% de diferença em relação ao ranqueamento ideal (Tabela 2), e o ranqueamento sem Fuzzy (Tabela 3) tem 33,3\% de diferença em relação ao mesmo ranqueamento (vide Tabela 5).

Tabela 5. Diferença dos métodos em relação ao Ranking Ideal

\begin{tabular}{|c|c|}
\hline Método de ranqueamento & Ranking Ideal \\
\hline Soma Ponderada sem Fuzzy & $33 \%$ \\
\hline Soma Ponderada com Fuzzy & $18 \%$ \\
\hline
\end{tabular}

Portanto, o método Soma Ponderada com Fuzzy está mais próximo do ranking ideal. Ressalta-se que os indicadores mais importantes (de maior peso) são do grupo 
áreas verdes (extração irregular de areia, argila ou cascalho e pesca irregular), e estes possuem ranqueamento mais próximo do ranking ideal quando se utiliza Fuzzy.

\subsection{Experimento 2}

Este experimento avalia a usabilidade do método Soma Ponderada-Fuzzy em comparação com o Soma Ponderada sem Fuzzy. Para isto, foi feito um experimento para verificar como seria o ajuste de pesos manualmente sem Fuzzy para gerar um ranqueamento ideal.

Para produzir resultado igual ao ideal, foi necessário ajustar os pesos de todos os aspectos ambientais. Vale ressaltar que o experimento necessitou de dezenas de tentativas de ajustes de pesos para obter resultado semelhante, isto é, não é tarefa trivial gerar o ranqueamento ideal com ajustes manuais de pesos. Portanto, o uso de Fuzzy aumenta a eficácia na redução do erro do modelo manual.

É importante destacar a percepção do gestor ambiental. Segundo o especialista, analista ambiental, biólogo especializado em planejamento urbano e gestão ambiental, a Lógica Fuzzy se mostrou mais eficaz na atribuição de pesos para os aspectos ambientais do que simplesmente atribuir um valor numérico a cada um deles. Para ele, o ranqueamento produzido pela aplicação de Fuzzy está mais próximo do ideal de acordo com os estudos atuais em gestão ambiental.

\section{Discussão}

A escolha de pesos por meio de variáveis linguísticas modificou o ranqueamento indicando que a interface com gestor ambiental pode afetar a acurácia do resultado. Além disso, a ferramenta agora permite ao gestor ambiental inferir pesos a partir da combinação das variáveis o que antes não era um processo claro. Agora é possível também determinar um peso específico para cada aspecto ambiental, que reflete a situação real em tomadas de decisão em gestão ambiental.

Vale ressaltar que o ranqueamento não foi feito simplesmente pela consideração dos indicadores mais denunciados pelos usuários. É considerada também a associação dos indicadores denunciados com outros presentes na base de dados para verificar a relação entre eles (confiança). Isto justifica o fato de o aspecto ambiental "Ar: Emissão de odores fétidos" estar à frente de "Solo: Extração irregular de areia, argila ou cascalho", pois Solo é um grupo de indicadores que tem maior peso que o grupo Ar, como mostra a Tabela 4.

Para este trabalho, duas dificuldades a serem superadas se destacam. A primeira delas é a obtenção de uma base de dados adequada ao estudo, isto é, uma base de dados volumosa, com denúncias da população de uma determinada cidade ou região urbana. Obter essa base pela aplicação web do ADGEPA não é trivial porque exige a necessidade de vários usuários para fazer várias denúncias ao longo de vários meses. Fazer a simulação dessas denúncias pode ser uma solução, mas também não é trivial porque é preciso que os dados simulados sejam coerentes, ou seja, é preciso evitar que uma denúncia sobre poluição de água contenha as coordenadas de um prédio em vez de um corpo d'água, por exemplo. Para superar o problema da simulação, já está sendo desenvolvido um simulador baseado em multiagentes. Por se tratar de uma técnica de Inteligência Artificial que permite desenvolver agentes computacionais com características de pró-atividade, reatividade, autonomia, e habilidades sociais, espera-se 
simular uma cidade ou região urbana populada por vários agentes para representar problemas socioambientais e cidadãos para fazer as denúncias.

A segunda dificuldade se refere à aplicação de pesos de indicadores pelo gestor. Embora a conversão de valores numéricos em valores linguísticos auxilie bastante o gestor, graças à Lógica Fuzzy, ainda é necessário bastante entendimento do modelo de negócio em questão. Para que um gestor possa aplicar bem os pesos aos grupos de indicadores socioambientais, é preciso que esteja bem claro para ele a relação custo/benefício agregada, haja vista que o ASD considera os aspectos socioambientais de forma sistêmica. Adicionalmente, é importante que o gestor e a população se desapeguem às ideias preconcebidas sobre combate a problemas socioambientais. Pode parecer que um aspecto foi desconsiderado ou pouco considerado ao se observar a ordenação de problemas oferecida pelo sistema; mas deve ser lembrado que a solução ou mitigação mais barata dos principais problemas pode se passar pela resolução ou mitigação de um ou mais problemas associados àquele em questão.

\section{Conclusão}

Pelo uso do método de decisão multicritério da Soma Ponderada-Fuzzy foi possível fazer ranqueamentos automáticos das opções disponíveis (as denúncias sobre os problemas socioambientais) mais próximos da realidade em gestão ambiental. O ASD considera os principais clamores dos usuários, os problemas socioambientais que têm maior associação entre eles (isso proporciona maior eficiência no uso de recursos financeiros públicos). Além disso, o ASD também permite o gestor entender o que é importante por meio de linguagem natural. Como resultado dos experimentos, a ponderação foi favorável aos aspectos ambientais de maior importância.

Portanto, o ASD é capaz de ranquear indicadores essenciais segundo à avaliação dos gestores ambientais, como por exemplo, os indicadores do grupo de "Áreas Verdes", conforme explicitado na Tabela 4, com maior facilidade e agilidade. Estes são fatores importantes na usabilidade da interface do sistema, que refletem na sua adoção e, principalmente, na acurácia dos resultados. Por outro lado, com o uso do ASD sem Fuzzy foi possível obter ranqueamentos mais distantes do que os desejados pelos gestores ambientais, como mostrado na Tabela 3.

Atualmente, há negociações para se aplicar o ASD em uma base de dados real da administração pública. Espera-se que o próximo trabalho futuro seja adaptar o ASD para trabalhar com os dados coletados pelo Cidadão Participativo, que é um projeto da Assembléia Legislativa do Estado de Goiás que tem como objetivo registrar ocorrências de problemas em municípios de Goiás. Outra possibilidade para realização de trabalhos futuros está relacionada à adaptação do ASD para trabalhar com dados gerados por redes sociais como o Twitter, uma vez que é possível extrair as coordenadas geográficas de uma possível denúncia e com a grande vantagem de ser uma rede social bastante utilizada.

\section{Agradecimentos}

Os autores agradecem às colaborações dos Profs. Ivanilton José de Oliveira, Philippe Caillou e Zach Lewkovicz. Também agradecem à Profa. Patrícia de Araújo Romão, Alex Pereira Maranhão, Mirelle Cristina Lima Silva e Carlos Henrique Barros Costa por suas participações passadas no trabalho. Esta pesquisa possui financiamento da Fundação de 
Amparo à Pesquisa de Goiás (FAPEG). Suportes adicionais individuais são fornecidos pelos programas de bolsas da Capes e do CNPq.

\section{Referências}

Afonso Amorim, L., Sebba Patto, V., Bulcão, R., and Gervásio Júnior, I. (2014). Suporte à decisão em gestão pública participativa: Um estudo de caso em gestão ambiental. In Ralha, C. G. and Adamatti, D. F., editors, Workshop em Computação Aplicada à Gestão do Meio Ambiente e Recursos Naturais (WCAMA), Brasília, DF, Brazil. SBC.

Almeida, A. T., Gomes, C. F. S., and Gomes, L. F. A. M. (2012). Tomada de Decisão Gerencial - Enfoque Multicritério. Atlas, 4 edition.

Chung, E.-S. and Kim, Y. (2014). Development of fuzzy multi-criteria approach to prioritize locations of treated wastewater use considering climate change scenarios. Journal of Environmental Management, 146(0):505 - 516.

Gamper, C. D. and Turcanu, C. (2007). On the governmental use of multi-criteria analysis. Ecological Economics, 62(2):298 - 307.

Gauthier, T. D. (2001). Detecting trends using spearman's rank correlation coefficient. Environmental Forensics, 2(4):359 - 362.

Larose, D. T. (2004). Discovering Knowledge in Data: An Introduction to Data Mining. Wiley-Interscience.

Lima Silva, M. C., Coelho, E. M. M., and Sebba Patto, V. (2013). Ambiente virtual de cenário urbano para simulação multiagentes - experiência do projeto adgepa. In X Encontro Nacional de Computação (ENACOMP-13), pages 97-104, Catalão, GO, Brasil. DCC-UFG.

Machado Junior, C., Mazzali, L., Souza, M. T. S. d., Furlaneto, C. J., and Prearo, L. C. (2013). A gestão dos recursos naturais nas organizações certificadas pela norma NBR ISO 14001. Produção, 23:41 - 51 .

Rowley, H. V., Peters, G. M., Lundie, S., and Moore, S. J. (2012). Aggregating sustainability indicators: Beyond the weighted sum. Journal of Environmental Management, 111(0):24 - 33.

Salafsky, N., Salzer, D., Ervin, J., Boucher, T., and Ostlie, W. (2013). Conventions for defining, naming, measuring, combining, and mapping threats in conservation - an initial proposal for a standard system. http://www.fosonline.org/.

Tulloch, V. J., Tulloch, A. I., Visconti, P., Halpern, B. S., Watson, J. E., Evans, M. C., Auerbach, N. A., Barnes, M., Beger, M., Chades, I., Giakoumi, S., McDonald-Madden, E., Murray, N. J., Ringma, J., and Possingham, H. P. (2015). Why do we map threats? linking threat mapping with actions to make better conservation decisions. Frontiers in Ecology and the Environment, 13(2):91-99.

Wibowo, S. and Deng, H. (2013). Consensus-based decision support for multicriteria group decision making. Computers e Industrial Engineering, 66(4):625 - 633.

Yeh, C.-H. and Xu, Y. (2013). Sustainable planning of e-waste recycling activities using fuzzy multicriteria decision making. Journal of Cleaner Production, 52(0):194 - 204. 
Ying, H. (2000). Fuzzy Control and Modeling: Analytical Foundations and Applications. Wiley-IEEE Press, 1st edition.

Ying, H., Ding, Y., Li, S., and Shao, S. (1999). Comparison of necessary conditions for typical takagi-sugeno and mamdani fuzzy systems as universal approximators. Systems, Man and Cybernetics, Part A: Systems and Humans, IEEE Transactions on, 29(5):508-514. 